\title{
MODELO DINÂMICO DE SIMULAÇÃO E OTIMIZAÇÃO DA FORÇA NORMAL DE REAÇÃO DO SOLO PARA UM MECANISMO DE CORTE BASAL ${ }^{1}$
}

\author{
CARLOS E. S. VOLPATO ${ }^{2}$, OSCAR A. BRAUNBECK ${ }^{3}$, CARLOS A. A. DE OLIVEIRA ${ }^{4}$
}

RESUMO: O modelo físico foi baseado no método de Newton-Euler, sendo o mesmo desenvolvido utilizando o programa computacional científico Mathematica ${ }^{\circledR}$. Realizaram-se várias simulações, nas quais se procurou obter a força normal de reação do solo variando velocidades de avanço $(0,69 ; 1,12$;

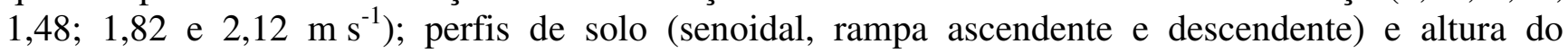
camalhão $(0,025$ e $0,05 \mathrm{~m})$. Após as simulações iniciais, o mecanismo foi otimizado utilizando o programa computacional científico Matlab ${ }^{\circledR}$, tendo como critério (função-objetivo) a minimização da força normal de reação do perfil $\left(\mathrm{F}_{\mathrm{N}}\right)$ e como variáveis de projeto os comprimentos das barras $\left(\mathrm{L}_{1 \mathrm{y}}, \mathrm{L}_{2}\right.$, $l_{3}$ e $L_{4}$ ), altura da operação (L7), o comprimento inicial da mola $\left(\mathrm{L}_{\mathrm{mo}}\right)$ e a constante elástica da mola $\left(\mathrm{k}_{\mathrm{t}}\right)$. A falta de robustez do mecanismo em relação à variável altura de operação foi contornada por meio do uso de mola com baixa rigidez e grande comprimento. Os resultados demonstraram que o mecanismo otimizado obteve desempenho de flutuação muito bom, em relação ao mecanismo inicial.

PALAVRAS-CHAVE: mecanismo de quatro barras, colheita mecânica, forragem.

\section{DYNAMIC MODEL OF SIMULATION AND OPTIMIZATION OF THE NORMAL FORCE OF SOIL REACTION FOR A MECHANISM OF BASE CUTTER}

\begin{abstract}
The physical model was based on the method of Newton-Euler. The model was developed by using the scientific computer program Mathematica ${ }^{\circledR}$. Several simulations where tried varying the progress speeds $\left(0.69 ; 1.12 ; 1.48 ; 1.82\right.$ and $\left.2.12 \mathrm{~m} \mathrm{~s}^{-1}\right)$; soil profiles (sinoidal, ascending and descending ramp) and height of the profile $(0.025$ and $0.05 \mathrm{~m})$ to obtain the normal force of soil reaction. After the initial simulations, the mechanism was optimized using the scientific computer program Matlab ${ }^{\circledR}$ having as criterion (function-objective) the minimization of the normal force of reaction of the profile (FN). The project variables were the lengths of the bars $\left(\mathrm{L}_{1 \mathrm{y}}, \mathrm{L}_{2}, \mathrm{l}_{3}\right.$ and $\left.\mathrm{L}_{4}\right)$, height of the operation $\left(\mathrm{L}_{7}\right)$, the initial length of the spring $\left(\mathrm{L}_{\mathrm{mo}}\right)$ and the elastic constant of the spring $\left(\mathrm{k}_{\mathrm{t}}\right)$. The lack of robustness of the mechanism in relation to the variable height of the operation was outlined by using a spring with low rigidity and large length. The results demonstrated that the mechanism optimized showed better flotation performance in relation to the initial mechanism.
\end{abstract}

KEYWORDS: four bar linkage, mechanical harvesting, forages.

\section{INTRODUÇÃO}

Segundo BRAUNBECK (1999), existem limitações no princípio operacional do cortador basal de discos rígidos das colhedoras de cana e forragens, gerando espaço tecnológico para o dimensionamento de mecanismos alternativos que diminuem ou evitam o recolhimento de solo e as perdas de matéria-prima durante a colheita que podem ser resolvidas por meio do emprego de

\footnotetext{
${ }^{1}$ Trabalho financiado pela FAPESP. Processo $n^{\circ}$ 99/04745-1

${ }^{2}$ Prof. Adjunto, Doutor, Departamento de Engenharia, UFLA, Lavras - MG, Fone: (0XX35) 3821.7962, volpato@ufla.br

${ }^{3}$ Prof. Ph.D, Faculdade de Engenharia Agrícola , UNICAMP, Campinas - SP, oscar@agr.unicamp.br

${ }^{4}$ Prof. Doutor, Curso de Agronomia da FESURV, Rio Verde - GO, caaoliv@agr.unicamp.br

Recebido pelo Conselho Editorial em: 2-7-2003

Aprovado pelo Conselho Editorial em: 20-10-2004
} 
ferramentas tecnológicas como modelagem virtual, combinada com técnicas de otimização de sistemas mecânicos que visam a soluções de engenharia alternativas e mais arrojadas.

A essência da modelagem encontra-se no estabelecimento, durante o processo de pesquisa, dos indicadores predominantes na determinação das relações funcionais fundamentais. Por isso, a modelagem das equações de movimento para um mecanismo de quatro barras consiste de um sistema de equações não-lineares das relações cinemáticas (deslocamentos, velocidades e acelerações) e dinâmicas (forças e momentos) do mecanismo (DOUGHTY, 1988). O uso de modelagem matemática no projeto de mecanismos é prática comum que permite o desenvolvimento de sistemas mecânicos com a redução de custos e tempos de projeto, e a otimização das características de desempenho. Esse uso abraça todos os aspectos do projeto e, em particular, o movimento. Através do estabelecimento de hipóteses simplificadas e da aplicação de leis físicas apropriadas, as equações de movimento são obtidas para representar os aspectos relevantes do comportamento do sistema mecânico a ser estudado (SANTOS, 2000).

Segundo PASQUINI JÚNIOR \& COSTA NETO (1996), no estudo de um problema de modelagem, a definição apropriada dos aspectos relevantes a serem considerados é um ponto muito importante. Assim, é necessário discutir qual deve ser o modelo utilizado, levando-se em consideração a complexidade desejada e sua aproximação com o modelo real. De maneira geral, o processo de modelagem matemática de um mecanismo de quatro barras segue os seguintes passos: $1^{\underline{0}}$ ) Descrever o modelo físico de um sistema, o qual contém os aspectos relevantes para o estudo desejado, com sua hipótese e simplificação; $2^{\underline{0}}$ ) Obter equações que descrevam matematicamente o comportamento do sistema; $3^{\underline{o}}$ ) Resolver as equações resultantes analiticamente ou numericamente com o objetivo de estimar o comportamento do sistema; $4^{-}$) Verificar os resultados do modelo por comparação com o comportamento do sistema real; $5^{\underline{0}}$ ) Modificar o modelo, se necessário, ou usá-lo para proposta de análise e projeto.

Atualmente, as principais técnicas de otimização tratam a síntese de mecanismos como um problema de programação não-linear, em que a solução ótima é obtida por meio da minimização de uma função-objetivo que é definida como o erro entre a curva desejada e a gerada por um ponto do acoplador. Pode-se dizer que a otimização consiste em um problema de programação matemática formulado da seguinte maneira: achar o valor das variáveis $X=\left[\mathrm{x}_{1}, \mathrm{x}_{2}, \ldots . . \mathrm{x}_{\mathrm{n}}\right]$, as quais minimizam ou maximizam uma determinada função, $f(x)$, do vetor $X$, sendo essa função denominada funçãoobjetivo. MURCIA (1998) otimizou um mecanismo articulado para o seguimento do perfil do solo em processos de colheita de grãos usando o software de otimização OPT 3.2., reduzindo o valor da força de reação do solo na ponta do garfo cortador de 24,4 para 10,8 $\mathrm{N}$ para um perfil senoidal de solo.

\section{MATERIAL E MÉTODOS}

\section{Análise cinemática e dinâmica (modelagem)}

As análises cinemáticas e dinâmicas do mecanismo, ilustrado pela Figura 1, foram feitas pelo método de Newton-Euler, conforme SANTOS (2000). Considerando cada barra do mecanismo como um vetor, é possível definir sua configuração geométrica instantânea pela equação vetorial de malha fechada, segundo DOUGHTY (1988), da qual resultam as eq.(1) e (4) que são as equações que definirão a geometria e a posição do mecanismo ao longo do perfil. 


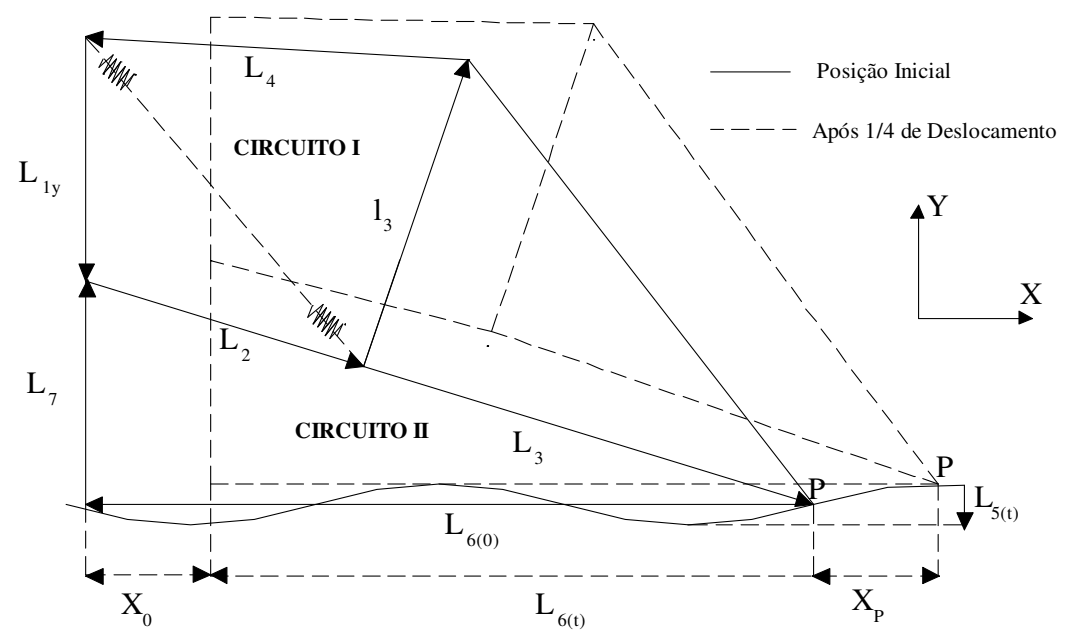

FIGURA 1. Representação das equações vetoriais de malha fechada para o mecanismo.

Circuito I: Define a geometria do mecanismo

$$
\begin{aligned}
& \overrightarrow{\mathrm{L}}_{1}+\overrightarrow{\mathrm{L}}_{2}+\overrightarrow{\mathrm{l}}_{3}+\overrightarrow{\mathrm{L}}_{4}=0 \\
& \mathrm{~L}_{2} \operatorname{Cos} \theta_{2}+1_{3} \operatorname{Cos} \theta_{3 \mathrm{a}}+\mathrm{L}_{4} \operatorname{Cos}_{4}=0 \\
& -\mathrm{L}_{1 \mathrm{Y}}+\mathrm{L}_{2} \operatorname{Sen} \theta_{2}+1_{3} \operatorname{Sen} \theta_{3 \mathrm{a}}+\mathrm{L}_{4} \operatorname{Sen} \theta_{4}=0
\end{aligned}
$$

Circuito II: Define a posição do mecanismo.

$$
\begin{aligned}
& \overrightarrow{\mathrm{L}}_{2}+\overrightarrow{\mathrm{L}}_{3}+\overrightarrow{\mathrm{L}}_{5}+\overrightarrow{\mathrm{L}}_{6}+\overrightarrow{\mathrm{L}}_{7}=0 \\
& \mathrm{~L}_{2} \operatorname{Cos} \theta_{2}+\mathrm{L}_{3} \operatorname{Cos} \theta_{3}-\mathrm{L}_{6}(\mathrm{t})=0 \\
& \mathrm{~L}_{2} \operatorname{Sen} \theta_{2}+\mathrm{L}_{3} \operatorname{Sen} \theta_{3}-\mathrm{L}_{5}(\mathrm{t})+\mathrm{L}_{7}=0 \\
& \theta_{3}-\theta_{3 \mathrm{a}}=\Delta \theta
\end{aligned}
$$

A solução das equações fornecerá a posição do mecanismo $\boldsymbol{\theta}_{\mathbf{2}}, \boldsymbol{\theta}_{\mathbf{3}}, \boldsymbol{\theta}_{\mathbf{4}} \mathbf{e} \mathbf{L}_{\mathbf{5}}(\mathbf{t})$. Analisando o circuito II da Figura 1, pode-se escrever a equação que definirá o posicionamento do disco cortador basal, em função do microrrelevo do solo. Essa será escrita em função do perfil que a superfície do solo seguirá durante a passagem do cortador basal. Nesse caso, em específico, foi verificado para o perfil de solo senoidal. A função que o descreve está mostrada pela eq.(8).

$$
Y_{P}(t)=A \operatorname{Sen}\left(\frac{2 \pi}{D s} X_{P}\right)
$$

em que,

$\mathrm{Y}_{\mathrm{P}}$ - cota correspondente à altura do perfil $\left(\mathrm{L}_{5}(\mathrm{t})\right), \mathrm{m}$;

A - metade da amplitude do solo, m;

Ds - distância entre sulcos, m;

$\mathrm{X}_{\mathrm{P}}$ - distância percorrida no plano horizontal $\left(\mathrm{X}_{0}-\Delta_{\mathrm{X}}\right), \mathrm{m}$;

$\mathrm{X}_{0}=\mathrm{Vt}, \mathrm{m}, \mathrm{e}$

$\Delta_{\mathrm{X}}=\overrightarrow{\mathrm{L}}_{6(0)}-\overrightarrow{\mathrm{L}}_{6}(\mathrm{t}), \mathrm{m}$.

Nas equações descritas, o perfil do solo será representado pela equação $\overrightarrow{\mathrm{L}}_{5(\mathrm{t})}$. 


$$
{ }_{\mathrm{I}} \overrightarrow{\mathrm{L}}_{5}=\mathrm{A} \operatorname{Sen}\left(\frac{2 \pi}{\mathrm{Ds}}\right)\left(\mathrm{X}_{0}-\Delta_{\mathrm{X}}\right)
$$

Assim sendo, temos:

$$
{ }_{\mathrm{I}} \overrightarrow{\mathrm{L}}_{5}=\operatorname{ASen}\left(\frac{2 \pi}{\mathrm{Ds}}\right)\left[(\mathrm{V} \mathrm{t})-\left(\overrightarrow{\mathrm{L}}_{6(0)}+\overrightarrow{\mathrm{L}}_{6}(\mathrm{t})\right)\right]
$$

Substituindo $\mathrm{L}_{5}$ na eq.(6), obtemos:

$$
\mathrm{L}_{2} \operatorname{Sen} \theta_{2}+\mathrm{L}_{3} \operatorname{Sen} \theta_{3}-\left[\mathrm{A} \operatorname{Sen}\left(\frac{2 \pi}{\mathrm{Ds}}\right) \mathrm{Vt}-\left(\overrightarrow{\mathrm{L}}_{6(0)}+\overrightarrow{\mathrm{L}}_{6}(\mathrm{t})\right)\right]+\mathrm{L}_{7}=0
$$

O vetor de velocidade angular foi definido como a primeira derivada do vetor de posição na base inercial em relação ao tempo. É importante ressaltar que a derivada desse vetor deve ser sempre feita no sistema de referência inercial. Assim sendo, as velocidades angulares serão obtidas derivando as eqs.(2), (3), (5), (6) e (7). O vetor de aceleração angular foi definido como a segunda derivada do vetor de posição na base inercial em relação ao tempo, também realizada no sistema de referência inercial. Assim sendo, as velocidades angulares serão obtidas pela segunda derivada das eq.(2), (3), (5), (6) e (7). As eq.(2), (3), (5), (6) e (7) formam um sistema de equações não-lineares com cinco equações e cinco incógnitas. A solução desse tem como resposta os valores de $\theta_{2}, \theta_{3}, \theta_{3 \mathrm{a}}, \theta_{4}$ e $\mathrm{L}_{5}(\mathrm{t})$. A resolução foi feita com auxílio do programa computacional Mathematica ${ }^{\circledR}$. O mesmo procedimento foi usado para resolver os sistemas de equações para determinar as velocidades e acelerações angulares e obter $\dot{\theta_{2}}, \dot{\theta_{3}}, \dot{\theta_{3 a}}, \dot{\theta_{4}}, \dot{L_{5(t)}}, \ddot{\theta_{2}}, \ddot{\theta_{3}}, \ddot{\theta_{3 a}}, \ddot{\theta}_{4} \mathrm{e} \ddot{\mathrm{L}}_{5(\mathrm{t})}$. A análise dinâmica permite determinar as reações nas articulações e a força de interação disco-solo, que constitui a função-objetivo a ser minimizada posteriormente. As equações de equilíbrio dinâmico, baseadas nas leis de Newton-Euler, são escritas para cada uma das barras usando o diagrama de corpo livre do mecanismo. Como produto final do método, obteremos as equações de movimento $\mathrm{e}$ as reações dinâmicas. $\mathrm{O}$ método Newton-Euler baseia-se, fundamentalmente, nas duas equações abaixo:

$$
\begin{aligned}
& \sum \mathrm{F}_{\mathrm{i}}=\mathrm{m}_{\mathrm{i}} \overrightarrow{\mathrm{a}}_{\mathrm{i}} \\
& \sum \mathrm{T}_{\mathrm{i}}=\mathrm{I}_{\mathrm{i}} \ddot{\theta}_{\mathrm{i}}
\end{aligned}
$$

em que, $F_{i}, T_{i}, m_{i}, I_{i}, \vec{a}_{i}$ e $\ddot{\theta}_{i}$ são as forças externas nas articulações, momentos externos, massa, momento de inércia no centro de gravidade e as acelerações de translação e rotação da barra $\mathbf{i}$, respectivamente. Assim sendo, com auxílio do diagrama de corpo livre da Figura 2, pode-se escrever o sistema de equações algébricas linear de equilíbrio dinâmico:

Equações obtidas do diagrama de corpo livre da barra 2:

$$
\begin{aligned}
& \sum \mathrm{F}_{2 \mathrm{X}}=\mathrm{F}_{\mathrm{OX}}+\mathrm{F}_{\mathrm{AX}}-\mathrm{F}_{\mathrm{M}} \operatorname{Cos}\left(\varepsilon \mathrm{O}-\mathrm{m}_{2} \mathrm{a}_{(\mathrm{x}) \mathrm{cm} 2}\right. \\
& \operatorname{Cos}\left(\varepsilon \mathrm{O}=\left(\mathrm{L}_{2} \operatorname{Cos} \theta_{2}\right) / \mathrm{L}_{\mathrm{m}}\right. \\
& \sum \mathrm{F}_{2 \mathrm{Y}}=\mathrm{F}_{\mathrm{OY}}+\mathrm{F}_{\mathrm{AY}}+\mathrm{F}_{\mathrm{M}} \operatorname{Sen}\left(\varepsilon \mathrm{e}-\mathrm{m}_{2} \mathrm{~g}-\mathrm{m}_{2} \mathrm{a}_{(\mathrm{y}) \mathrm{cm} 2}\right. \\
& \operatorname{Sen}\left(\varepsilon \mathrm{e}=\left(\mathrm{L}_{1 \mathrm{y}}+\mathrm{L}_{2} \operatorname{Sen} \theta_{2}\right) / \mathrm{L}_{\mathrm{m}}\right.
\end{aligned}
$$




$$
\begin{aligned}
\sum \mathrm{T}_{2}= & -\mathrm{F}_{\mathrm{OX}}\left(\frac{\mathrm{L}_{2}}{2}\right) \operatorname{Cos}\left(\theta_{2}\right)-\mathrm{F}_{\mathrm{OY}}\left(\frac{\mathrm{L}_{2}}{2}\right) \operatorname{Sen}\left(\theta_{2}\right)+\mathrm{F}_{\mathrm{AX}}\left(\frac{\mathrm{L}_{2}}{2}\right) \operatorname{Cos}\left(\theta_{2}\right)+\mathrm{F}_{\mathrm{AY}}\left(\frac{\mathrm{L}_{2}}{2}\right) \operatorname{Sen}\left(\theta_{2}\right)+ \\
& \mathrm{F}_{\mathrm{M}} \operatorname{Sen}\left(\varepsilon \mathrm{e}\left(\frac{\mathrm{L}_{2}}{2}\right) \operatorname{Cos}\left(\theta_{2}\right)-\mathrm{F}_{\mathrm{M}} \operatorname{Cos}\left(\varepsilon \mathrm{O}\left(\frac{\mathrm{L}_{2}}{2}\right) \operatorname{Sen}\left(\theta_{2}\right)-\mathrm{I}_{2 \mathrm{ZZ}} \ddot{\theta}_{2}\right.\right.
\end{aligned}
$$

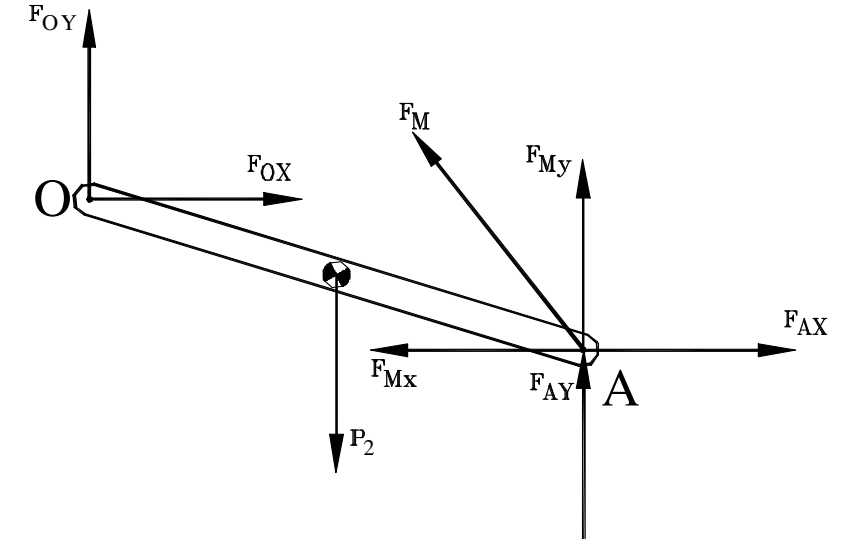

Barra 2

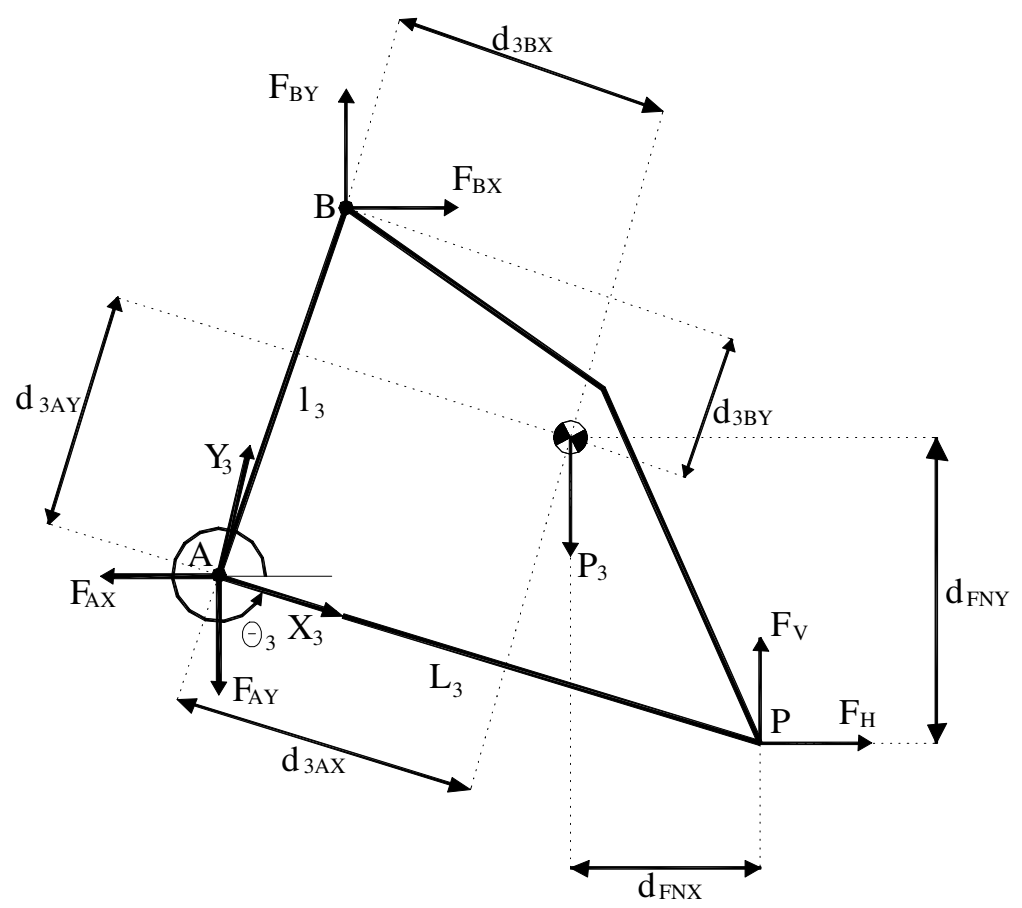

Barra 3

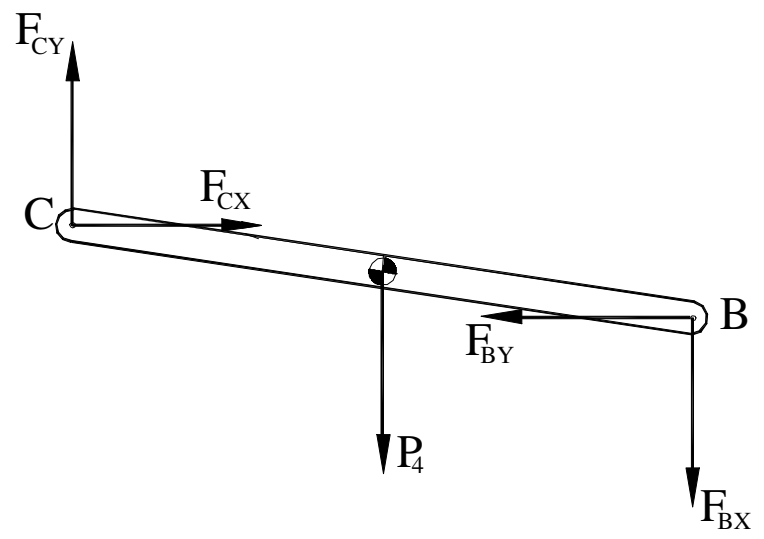

Barra 4

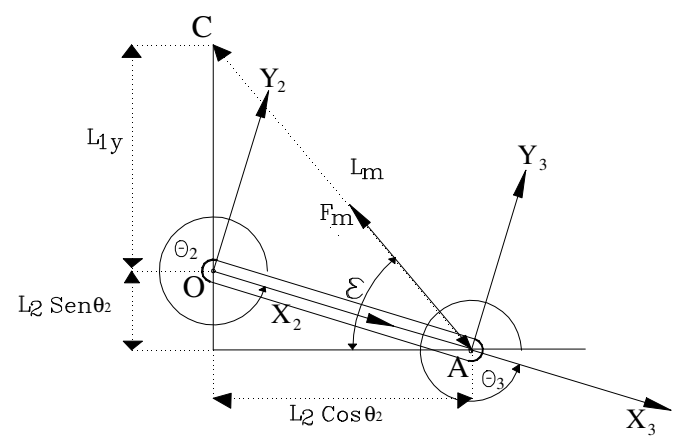

Ângulo Épsilon

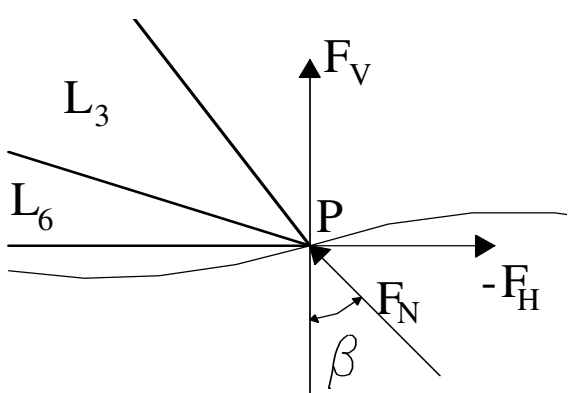

Ângulo Beta

FIGURA 2. Digrama de corpo das barras do mecanismo e definição dos ângulos $\beta$ e $\varepsilon$.

Equações obtidas do diagrama de corpo livre da barra 3:

$$
\begin{aligned}
& \sum \mathrm{F}_{3 \mathrm{X}}=-\mathrm{F}_{\mathrm{AX}}+\mathrm{F}_{\mathrm{BX}}-\mathrm{F}_{\mathrm{N}} \operatorname{Sen}\left(\beta \mathrm{e}-\mathrm{m}_{2} \mathrm{a}_{(\mathrm{x}) \mathrm{cm} 3}\right. \\
& \operatorname{Sen} \beta=\left(-\mathrm{F}_{\mathrm{H}} / \mathrm{F}_{\mathrm{N}}\right) \\
& \sum \mathrm{F}_{3 \mathrm{Y}}=-\mathrm{F}_{\mathrm{AY}}+\mathrm{F}_{\mathrm{BY}}+\mathrm{F}_{\mathrm{N}} \operatorname{Cos}\left(\beta \mathrm{o}-\mathrm{m}_{2} \mathrm{~g}-\mathrm{m}_{2} \mathrm{a}_{(\mathrm{y}) \mathrm{cm} 3}\right.
\end{aligned}
$$




$$
\begin{aligned}
& \operatorname{Cos} \beta=\left(\mathrm{F}_{\mathrm{V}} / \mathrm{F}_{\mathrm{N}}\right) \\
& \sum \mathrm{T}_{3}=-\mathrm{F}_{\mathrm{AX}} \mathrm{d}_{3 A Y}+\mathrm{F}_{\mathrm{AY} \mathrm{I}} \mathrm{d}_{3 \mathrm{AX}}-\mathrm{F}_{\mathrm{BX} \text { I }} \mathrm{d}_{3 A Y}-\mathrm{F}_{\mathrm{BY} I} \mathrm{~d}_{3 A X}-\mathrm{F}_{\mathrm{N}} \operatorname{Sen}(\beta)_{\mathrm{I}} \mathrm{d}_{\mathrm{FNY}}+ \\
& \mathrm{F}_{\mathrm{N}} \operatorname{Cos}(\beta)_{\mathrm{I}} \mathrm{d}_{\mathrm{FNX}}-\mathrm{I}_{3 Z Z} \ddot{\theta}_{3}
\end{aligned}
$$

Equações obtidas do diagrama de corpo livre da barra 4:

$$
\begin{aligned}
\sum \mathrm{F}_{4 \mathrm{X}} & =\mathrm{F}_{\mathrm{CX}}-\mathrm{F}_{\mathrm{BX}}-\mathrm{m}_{4} \mathrm{a}_{(\mathrm{x}) \mathrm{cm} 4} \\
\sum \mathrm{F}_{4 \mathrm{Y}} & =\mathrm{F}_{\mathrm{CY}}-\mathrm{F}_{\mathrm{BY}}-\mathrm{m}_{4} \mathrm{~g}-\mathrm{m}_{4} \mathrm{a}_{(\mathrm{y}) \mathrm{cm} 4} \\
\sum \mathrm{T}_{4}= & -\mathrm{F}_{\mathrm{CX}}\left(\frac{\mathrm{L}_{4}}{2}\right) \operatorname{Sen}\left(\theta_{4}\right)-\mathrm{F}_{\mathrm{CY}}\left(\frac{\mathrm{L}_{4}}{2}\right) \operatorname{Cos}\left(\theta_{4}\right)-\mathrm{F}_{\mathrm{BX}}\left(\frac{\mathrm{L}_{4}}{2}\right) \operatorname{Sen}\left(\theta_{4}\right)- \\
& \mathrm{F}_{\mathrm{BY}}\left(\frac{\mathrm{L}_{4}}{2}\right) \operatorname{Cos}\left(\theta_{4}\right)-\mathrm{I}_{4 \mathrm{ZZ}} \ddot{\theta}_{4}
\end{aligned}
$$

Do diagrama de corpo livre, obtém-se um sistema de equações lineares com nove equações e nove incógnitas que foi mostrado acima. Esse sistema pode ser escrito sob a forma matricial para obter as nove incógnitas $F_{O x}, F_{O y}, F_{A x}, F_{A y}, F_{B x}, F_{B y}, F_{C x}, F_{C y}$ e $F_{N}$ em função do tempo, em que:

$$
[\mathrm{L}]\left[\mathrm{F}_{\mathrm{B}}\right]=\left[\mathrm{F}_{\mathrm{I}}\right]
$$

em que,

[L] - matriz quadrada dos parâmetros de posição e propriedades das barras;

$\left[\mathrm{F}_{\mathrm{B}}\right]$ - vetor coluna das forças nas articulações e na ponta do disco (incógnitas), e

$\left[\mathrm{F}_{\mathrm{I}}\right]$ - vetor coluna das forças e torques conhecidos.

\section{Simulações e otimização}

A partir da modelagem matemática do mecanismo cortador basal, desenvolveu-se um programa utilizando a linguagem simbólica do software de computação científica Mathematica ${ }^{\circledR}$ para a simulação do mecanismo, possibilitando a avaliação de desempenho por meio dos valores máximos e mínimos da força normal de interação solo-mecanismo. Para todas as simulações realizadas, foram utilizadas as seguintes variáveis para verificar o desempenho de flutuação do mecanismo: perfis (senoidal, rampa ascendente e rampa descendente), amplitude do perfil (0,025 $\mathrm{m}$ e 0,05 $\mathrm{m}$ ), velocidade de avanço da máquina $\left(0,68 \mathrm{~m} \mathrm{~s}^{-1} ; 1,12 \mathrm{~m} \mathrm{~s}^{-1} ; 1,48 \mathrm{~m} \mathrm{~s}^{-1} ; 1,82 \mathrm{~m} \mathrm{~s}^{-1} \mathrm{e} 2,20 \mathrm{~m} \mathrm{~s}^{-1}\right)$. A força normal de reação do solo ao longo do período de tempo foi definida como sendo a função-objetivo a ser minimizada. A forma utilizada no processo de otimização foi minimização da amplitude ao quadrado. O processo de otimização desenvolveu-se por meio do uso do programa computacional científico Matlab®.

$$
\mathrm{F}=\left(\mathrm{F}_{\mathrm{MAX}}-\mathrm{F}_{\mathrm{MIM}}\right)^{2}
$$

em que,

$\mathrm{F}$ - função-objetivo a ser minimizada, $\mathrm{N}$;

$\mathrm{F}_{\mathrm{MAX}}$ - valor máximo registrado pela simulação, $\mathrm{N}, \mathrm{e}$

$\mathrm{F}_{\mathrm{MIM}}$ - valor mínimo registrado pela simulação, N.

Os valores das forças fornecidos à função-objetivo para cálculo dos valores de máximo e mínimo foram gerados pelo $M a t l a b \AA$ dentro de um programa de apoio ao algoritmo principal. O processo de otimização foi feito com auxílio do módulo Toolbox-Optim do programa computacional Matlab. O programa principal utiliza a função "Constr" do Matlab para minimização com restrições. Esse processo contempla restrições de espaço, de trajetória, bem como restrições relativas à própria função- 
objetivo. Para isso, elas foram normalizadas e adimensionalizadas para uniformizar a magnitude das grandezas físicas, de onde se obtiveram os seguintes parâmetros:

- Constante da mola $\left(\mathrm{k}_{\mathrm{t}}\right): \mathrm{Kt} \geq 0 \quad[\mathrm{~N} / \mathrm{m}]$;

- Força normal de reação do perfil $\left(\mathrm{F}_{\mathrm{N}}\right): \mathrm{F}_{\mathrm{N}}>0 \quad[\mathrm{~N}]$;

- Trajetória (angular) ( $\left.\theta_{3}\right): 6,0 \leq \theta_{3} \leq 6,2$ [radianos];

- Comprimento inicial da mola $\left(\mathrm{L}_{\mathrm{mo}}\right): \mathrm{Lmo}>0 \quad[\mathrm{~m}]$;

- Altura entre ponto de contato e articulação $\mathrm{L}_{1} / \mathrm{L}_{2}\left(\mathrm{~L}_{7}\right): \mathrm{L}_{7}>0$;

- Comprimento das Barras (m):

- Limite inferior (vlb): $0,0\left[\begin{array}{lllll}\mathrm{L}_{1 \mathrm{y}} & \mathrm{L}_{2} & \mathrm{l}_{3} & \mathrm{~L}_{3} & \mathrm{~L}_{4}\end{array}\right]$;

- Limite superior (vub): 2,0 $\left[\begin{array}{lllll}\mathrm{L}_{1 \mathrm{y}} & \mathrm{L}_{2} & \mathrm{l}_{3} & \mathrm{~L}_{3} & \mathrm{~L}_{4}\end{array}\right]$.

\section{RESULTADOS E DISCUSSÃO}

As simulações feitas no Matlab ${ }^{\circledR}$ encontram-se ilustradas nas Figuras 3 a 6 . Os resultados da força normal de reação do perfil expressos em força máxima, mínima e no valor de sua amplitude, para todas as simulações realizadas, encontram-se nas Tabelas 1 a 3.

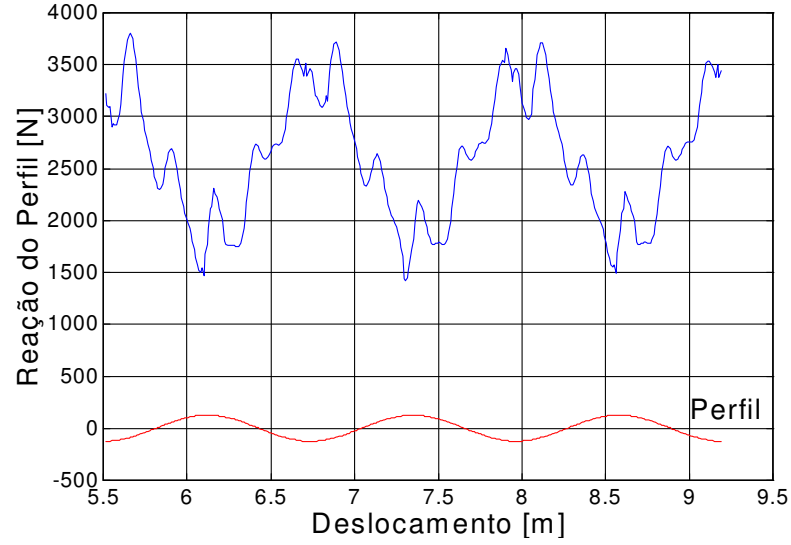

FIGURA 3. Simulação de $\mathrm{F}_{\mathrm{N}}$ no Matlab para perfil
senoidal de $0,025 \mathrm{~m}$ e velocidade de $1,82 \mathrm{~m} \mathrm{~s}^{-1}$

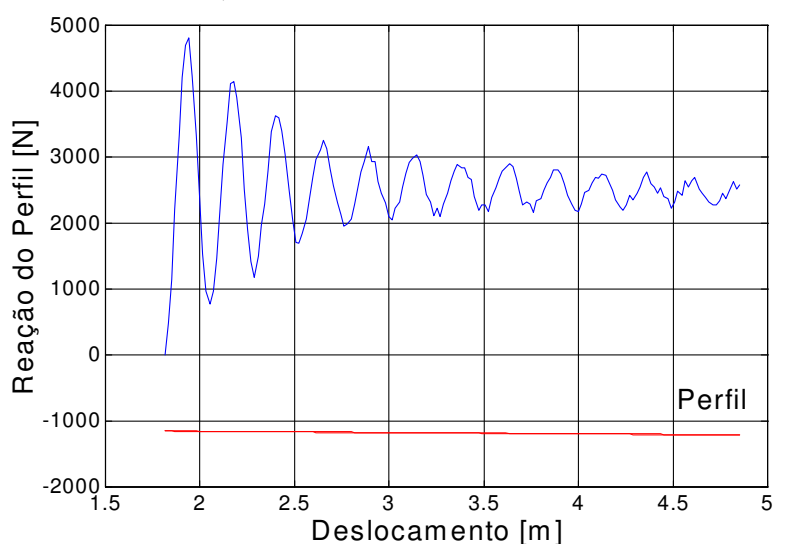

FIGURA 5. Simulação de $\mathrm{F}_{\mathrm{N}}$ no Matlab para perfil rampa descendente de $0,025 \mathrm{~m} \quad \mathrm{e}$ velocidade de $1,82 \mathrm{~m} \mathrm{~s}^{-1}$

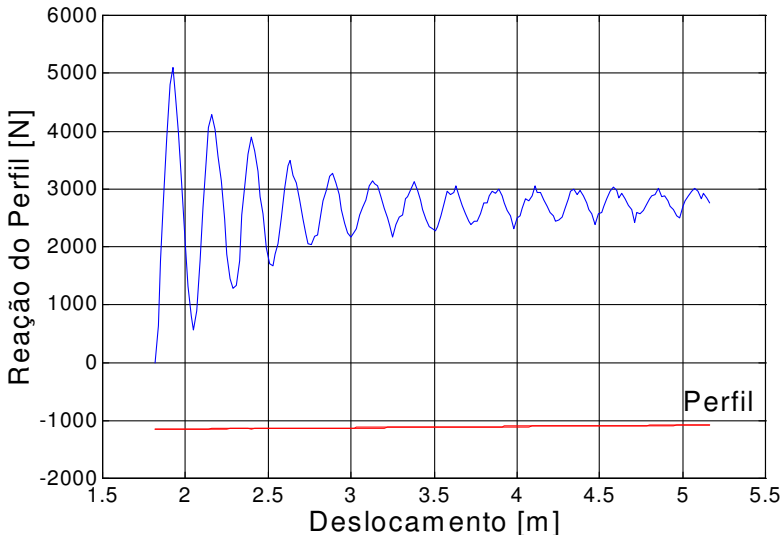

FIGURA 4. Simulação de $\mathrm{F}_{\mathrm{N}}$ no Matlab para perfil rampa ascendente de $0,025 \mathrm{~m}$ e velocidade de $1,82 \mathrm{~m} \mathrm{~s}^{-1}$

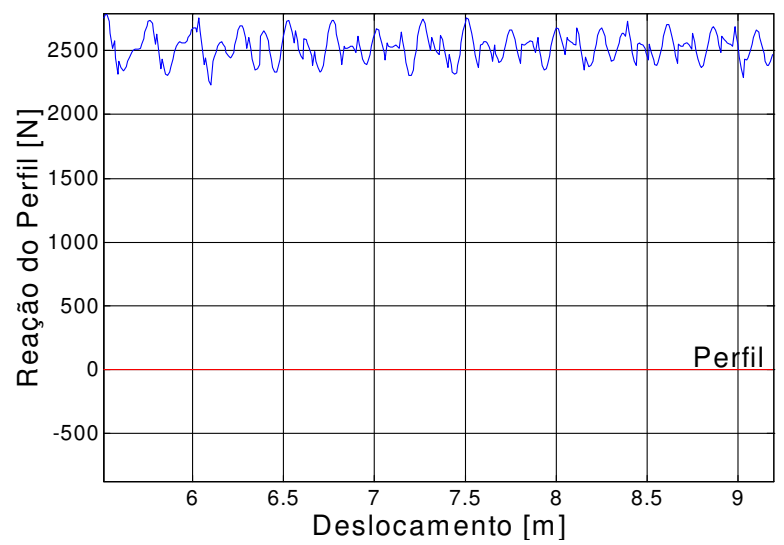

FIGURA 6. Simulação de $F_{N}$ no Matlab para perfil com amplitude zero e velocidade de $1,82 \mathrm{~m} \mathrm{~s}^{-1}$ 
Analisando as Tabelas 1 a 3, pode-se observar que a amplitude do perfil (A) e a velocidade de deslocamento da máquina (v) tem influência significativa sobre força normal de reação do perfil $\left(\mathrm{F}_{\mathrm{N}}\right)$, quando se tem em avaliação o perfil senoidal. Esse comportamento se dá pelo fato de essa amplitude de força corresponder à componente inercial que movimenta a massa do cortador de base, ou seja, surge da aceleração dessa massa ao qual é proporcional à amplitude do perfil e à velocidade angular. Para os perfis em rampa, tanto ascendentes quanto descendentes, a velocidade de avanço da máquina demonstrou pouca ou quase nenhuma influência sobre a força normal de reação do perfil.

TABELA 1. Simulação da força de reação normal para perfil do tipo senoidal.

\begin{tabular}{ccccccc}
\hline & \multicolumn{3}{c}{$\mathrm{a}=0,05(\mathrm{~m})$} & \multicolumn{3}{c}{$\mathrm{a}=0,025(\mathrm{~m})$} \\
\cline { 2 - 7 } $\left.\mathrm{v}^{-1} \mathrm{~m} \mathrm{~s}^{-1}\right)$ & $\mathrm{F}_{\mathrm{N} \max }$ & $\mathrm{F}_{\mathrm{N} \min }$ & $\mathrm{A}$ & $\mathrm{F}_{\mathrm{N} \max }$ & $\mathrm{F}_{\mathrm{N} \min }$ & $\mathrm{A}$ \\
& $(\mathrm{N})$ & $(\mathrm{N})$ & $(\mathrm{N})$ & $(\mathrm{N})$ & $(\mathrm{N})$ & $(\mathrm{N})$ \\
\hline 0,69 & $2.512,41$ & $1.690,36$ & 822,05 & $2.304,68$ & $1.892,87$ & 411,81 \\
1,12 & $2.849,50$ & $1.358,36$ & $1.491,14$ & $2.471,38$ & $1.725,60$ & 745,78 \\
1,48 & $3.258,50$ & 959,337 & $2.299,16$ & $2.672,03$ & $1.524,45$ & $1.147,58$ \\
1,82 & $3.735,23$ & 481,171 & $3.272,06$ & $2.912,73$ & $1.283,33$ & $1.629,40$ \\
2,12 & $4.280,42$ & $-22,466$ & $4.302,88$ & $3.166,29$ & $1.029,32$ & $2.136,97$ \\
\hline
\end{tabular}

a - amplitude do perfil senoidal e A - amplitude da força normal de reação do perfil

TABELA 2. Simulação da força de reação normal para perfil do tipo rampa ascendente.

\begin{tabular}{ccccccc}
\hline & \multicolumn{3}{c}{$\mathrm{a}=0,05(\mathrm{~m})$} & \multicolumn{3}{c}{$\mathrm{a}=0,025(\mathrm{~m})$} \\
\cline { 2 - 7 } $\left.\mathrm{v} \mathrm{m} \mathrm{s}^{-1}\right)$ & $\mathrm{F}_{\mathrm{N} \max }$ & $\mathrm{F}_{\mathrm{N} \min }$ & $\mathrm{A}$ & $\mathrm{F}_{\mathrm{N} \max }$ & $\mathrm{F}_{\mathrm{N} \min }$ & $\mathrm{A}$ \\
& $(\mathrm{N})$ & $(\mathrm{N})$ & $(\mathrm{N})$ & $(\mathrm{N})$ & $(\mathrm{N})$ & $(\mathrm{N})$ \\
\hline 0,69 & $2.337,63$ & $2.126,57$ & 211,06 & $2.204,11$ & $2.099,51$ & 104,60 \\
1,12 & $2.312,02$ & $2.102,94$ & 209,08 & $2.204,26$ & $2.100,07$ & 104,19 \\
1,48 & 2.313 .50 & $2.105,03$ & 208,47 & $2.204,76$ & $2.100,67$ & 104,09 \\
1,82 & $2.315,06$ & $2.107,44$ & 207,62 & $2.205,23$ & $2.101,35$ & 103,88 \\
2,12 & $2.316,99$ & $2.109,92$ & 207,07 & $2.205,81$ & $2.102,03$ & 103,78 \\
\hline
\end{tabular}

a - amplitude do perfil senoidal e A - amplitude da força normal de reação do perfil

TABELA 3. Simulação da força de reação normal para perfil do tipo rampa descendente.

\begin{tabular}{ccccccc}
\hline & \multicolumn{3}{c}{$\mathrm{a}=0,05(\mathrm{~m})$} & \multicolumn{3}{c}{$\mathrm{a}=0,025(\mathrm{~m})$} \\
\cline { 2 - 7 } $\mathrm{v}\left(\mathrm{m} \mathrm{s}^{-1}\right)$ & $\mathrm{F}_{\mathrm{N} \max }$ & $\mathrm{F}_{\mathrm{N} \min }$ & $\mathrm{A}$ & $\mathrm{F}_{\mathrm{N} \max }$ & $\mathrm{F}_{\mathrm{N} \min }$ & $\mathrm{A}$ \\
& $(\mathrm{N})$ & $(\mathrm{N})$ & $(\mathrm{N})$ & $(\mathrm{N})$ & $(\mathrm{N})$ & $(\mathrm{N})$ \\
\hline 0,69 & $2.097,41$ & $1.894,06$ & 202,81 & $2.097,69$ & $1.994,90$ & 102,79 \\
1,12 & $2.098,33$ & $1.894,17$ & 204,16 & $2.097,82$ & $1.994,91$ & 102,91 \\
1,48 & 2.099 .58 & $1.894,13$ & 205,45 & $2.098,06$ & $1.994,73$ & 103,33 \\
1,82 & $2.101,18$ & $1.894,75$ & 206,43 & $2.098,38$ & $1.994,82$ & 103,56 \\
2,12 & $2.102,91$ & $1.894,44$ & 208,47 & $2.098,75$ & $1.994,98$ & 103,77 \\
\hline
\end{tabular}

a - amplitude do perfil senoidal e A - amplitude da força normal de reação do perfil

A amplitude do perfil teve muita influência sobre a amplitude da força normal de reação do perfil, visto que, quando seu valor é dobrado, isto é, passando de 0,025 para 0,05 m, verifica-se que o valor da amplitude da força normal de reação do perfil também fica dobrado, em todos os casos estudados, independentemente do valor da velocidade de deslocamento da máquina e do tipo de perfil.

A velocidade de avanço da máquina demonstrou ter influência sobre a amplitude da força normal de reação do perfil somente para o caso do perfil senoidal, em que se observou variação de 423,32\% para amplitude do perfil de $0,05 \mathrm{~m}$ e $418,92 \%$ para amplitude do perfil de 0,025 $\mathrm{m}$. Para os casos dos 
perfis em rampa ascendente e descendente, essa variação é de menos de $1 \%$ em todos os casos, independentemente da amplitude do perfil. Isso se justifica pelo fato de não haver aceleração vertical nesses tipos de perfil. O perfil senoidal apresentou-se como o mais crítico, uma vez que sofre influência de todas as variáveis avaliadas; apresenta os maiores valores de amplitude da força, e é aquele que, para algumas situações, o mecanismo de corte basal apresenta dificuldades de flutuação, como no caso da velocidade de deslocamento de $2,12 \mathrm{~m} \mathrm{~s}^{-1}$ com amplitude do perfil de 0,05 $\mathrm{m}$.

Em termos de acompanhamento do perfil pelo mecanismo inicial, o mesmo demonstrou excelente desempenho para todos os perfis avaliados, conforme registram as Figuras 3 a 6 . Para o perfil senoidal, observa-se esse acompanhamento do perfil pelo mecanismo inicial, uma vez que a força máxima é registrada no ponto máximo da senóide e a mínima no ponto mínimo da senóide, isto é, perfil e força estão em fase. Para os perfis em rampa, o mesmo desempenho é verificado, pois se observa que a força aumenta à medida que o mecanismo sobe a rampa e decresce à medida que o mecanismo desce a rampa. Analisando os resultados obtidos pelas simulações com o mecanismo inicial, decidiu-se utilizar as condições mais críticas como base de otimização, partindo-se da premissa que uma vez minimizada a força de reação normal do perfil para a pior condição, as outras condições acompanhariam esse processo. Assim sendo, o processo de otimização teve como base o perfil senoidal, com amplitude do perfil de $0,05 \mathrm{~m}$ e velocidade de avanço da máquina de $2,12 \mathrm{~m} \mathrm{~s}^{-1}$.

As simulações da força normal de reação do perfil para a velocidade de deslocamento de $2,12 \mathrm{~m} \mathrm{~s}^{-1}$ e perfil senoidal de amplitude de $0,05 \mathrm{~m}$ para o mecanismo não-otimizado e otimizado com e sem amortecimento estão ilustradas nas Figuras 7 e 8, respectivamente.

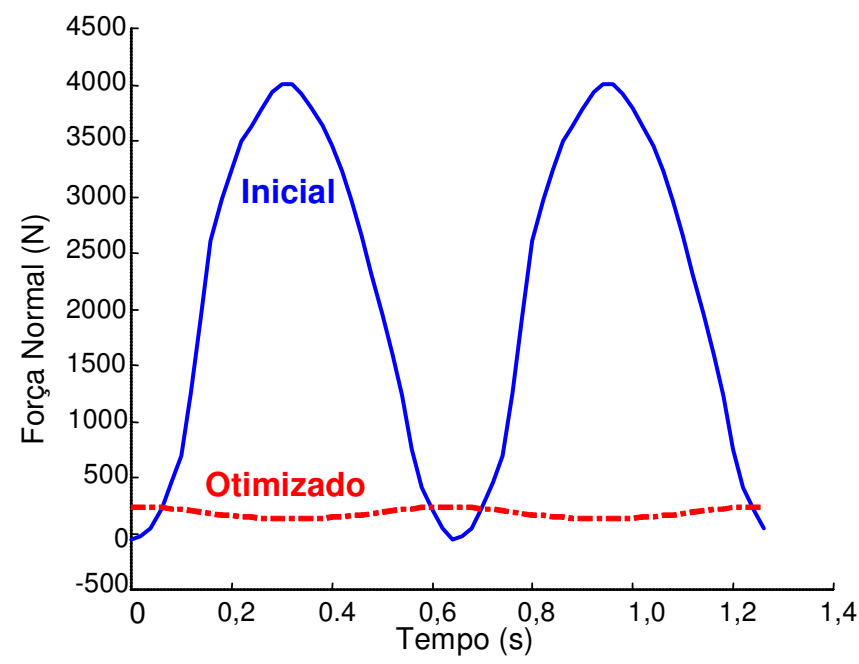

FIGURA 7. Força normal de reação do perfil $\left(\mathrm{F}_{\mathrm{N}}\right)$ para os mecanismos inicial e otimizado com mola.

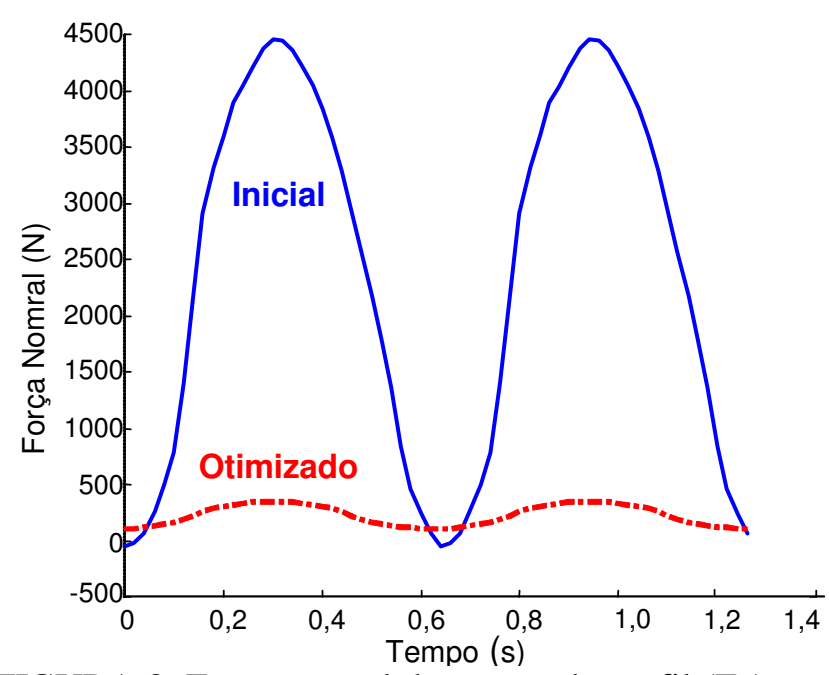

FIGURA 8. Força normal de reação do perfil $\left(\mathrm{F}_{\mathrm{N}}\right)$ para os mecanismos inicial e otimizado com mola e amortecimento.

Analisando as Tabelas 4 e 5, pode-se verificar que, após o processo de otimização, os parâmetros de projetos que mais sofreram alteração em suas dimensões foram a amplitude da articulação inferior até o ponto de contato com o perfil $\left(\mathrm{L}_{7}\right)$, a constante elástica da mola $\left(\mathrm{k}_{\mathrm{t}}\right)$ e a constante de amortecimento (c).

Comparando-se os mecanismos inicial e otimizado das Figuras 7 e 8, verifica-se que o processo de otimização das variáveis de projeto reduziu de forma significativa o valor da força normal de reação do perfil. Essa comparação entre os mecanismos inicial e otimizado, com e sem amortecimento, em termos de valor máximo, mínimo e amplitude da força pode ser visualizada na Tabela 6. 
TABELA 4. Variáveis de projeto antes e depois do processo de otimização apenas com mola.

\begin{tabular}{cccccccc}
\hline & $\mathrm{L}_{1 \mathrm{Y}}(\mathrm{m})$ & $\mathrm{L}_{2}(\mathrm{~m})$ & $\mathrm{l}_{3}(\mathrm{~m})$ & $\mathrm{L}_{4}(\mathrm{~m})$ & $\mathrm{L}_{7}(\mathrm{~m})$ & $\mathrm{L}_{\mathrm{mi}}(\mathrm{m})$ & $\mathrm{k}_{\mathrm{t}}\left(\mathrm{N} \mathrm{m}^{-1}\right)$ \\
\hline Inicial & 0,600 & 0,716 & 0,796 & 0,947 & 0,544 & 0,700 & 15.000 \\
Otimizado & 0,770 & 0,502 & 1,051 & 0,782 & 0,168 & 0,819 & 103.499 \\
Var. $(\%)$ & $22(\uparrow)$ & $29,9(\downarrow)$ & $24,26(\uparrow)$ & $17,42(\downarrow)$ & $323(\downarrow)$ & $12,5(\uparrow)$ & $690(\uparrow)$ \\
\hline
\end{tabular}

$(\uparrow)$ Aumento na dimensão do parâmetro avaliado; $(\downarrow)$ Diminuição na dimensão do parâmetro avaliado.

TABELA 5. Variáveis de projeto antes e depois do processo de otimização com mola e amortecedor.

\begin{tabular}{ccccccccc}
\hline & $\mathrm{L}_{1 \mathrm{Y}}(\mathrm{m})$ & $\mathrm{L}_{2}(\mathrm{~m})$ & $\mathrm{l}_{3}(\mathrm{~m})$ & $\mathrm{L}_{4}(\mathrm{~m})$ & $\mathrm{L}_{\mathrm{mi}}(\mathrm{m})$ & $\mathrm{L}_{7}(\mathrm{~m})$ & $\mathrm{c}\left(\mathrm{N} \mathrm{s} \mathrm{m}^{-1}\right)$ & $\mathrm{k}_{\mathrm{t}}\left(\mathrm{N} \mathrm{m}^{-1}\right)$ \\
\hline Inicial & 0,600 & 0,716 & 0,796 & 0,947 & 0,700 & 0,544 & $3.000,0$ & $15.000,0$ \\
Otimizado & 0,764 & 0,545 & 1,022 & 0,823 & 0,821 & 0,235 & 302,13 & $112.422,3$ \\
Var. $(\%)$ & $21(\uparrow)$ & $22,6(\downarrow)$ & $22,1(\uparrow)$ & $13,1(\downarrow)$ & $14,7(\uparrow)$ & $56,8(\downarrow)$ & $993(\downarrow)$ & $749,5(\uparrow)$ \\
\hline
\end{tabular}

$(\uparrow)$ Aumento na dimensão do parâmetro avaliado ; $(\downarrow)$ Diminuição na dimensão do parâmetro avaliado.

TABELA 6. Variação da força normal de reação do perfil, em [N], pelos mecanismos inicial e otimizado.

\begin{tabular}{ccccccc}
\hline & \multicolumn{3}{c}{ Mola } & \multicolumn{3}{c}{ Mola e Amortecedor } \\
\cline { 2 - 7 } & $\mathrm{F}_{\mathrm{N}} \mathrm{m}_{\mathrm{ax}}$ & $\mathrm{F}_{\mathrm{N}} \mathrm{m}_{\mathrm{in}}$ & $\mathrm{A}$ & $\mathrm{F}_{\mathrm{N}} \mathrm{m}_{\mathrm{ax}}$ & $\mathrm{F}_{\mathrm{N}} \mathrm{m}_{\text {in }}$ & $\mathrm{A}$ \\
\hline Inicial & $4.250,33$ & 10,09 & $4.240,24$ & $4.612,29$ & $-5,54$ & $4.617,83$ \\
Otimizado & 237,13 & 136,65 & 100,48 & 348,87 & 122,76 & 226,11 \\
Var. (\%) & $1.792,5(\downarrow)$ & $1.360(\uparrow)$ & $4.220(\downarrow)$ & $1.322(\downarrow)$ & $128,3(\uparrow)$ & $2.042(\downarrow)$ \\
\hline
\end{tabular}

( $\uparrow$ ) Aumento na força normal de reação do perfil; ( $\downarrow$ ) Diminuição na força normal de reação do perfil.

Observa-se, na Tabela 6, que o mecanismo sem amortecimento teve sua amplitude da força reduzida em $4.220 \%$, ao passo que, com a presença de amortecedor, essa redução foi de $2.042,03 \%$. Para o perfil senoidal, a amplitude é muito mais importante do que valores máximos ou mínimos analisados isoladamente.

Dessa forma, optou-se em adotar o mecanismo apenas com mola, apesar de o mecanismo com mola e amortecimento ter dado valores da força mínima menor. Outro fator importante é que o processo de otimização praticamente eliminou o amortecimento do sistema, reduzindo em aproximadamente $990 \%$ o coeficiente (c) inicial, conforme se pôde verificar na Tabela 5.

Em relação ao acompanhamento do perfil, observa-se que, para o mecanismo inicial, a força acompanha o perfil em fase, ou seja, a força máxima é registrada no ponto máximo da senóide do perfil, com ou sem amortecimento. Os mecanismos otimizados sem amortecimento evidenciam um ângulo de fase de aproximadamente $180^{\circ}$ entre o perfil e a força de reação. Isso surge como conseqüência da baixa freqüência natural do mesmo $(\leq 0,3 \mathrm{~Hz})$. Já para o mecanismo com mola e amortecimento, o perfil e a força de reação apresentam-se em fase, devido à ação do amortecedor.

\section{CONCLUSÕES}

O mecanismo de quatro barras representa um recurso construtivamente simples e estruturalmente robusto para a sustentação do cortador de base; no entanto, a complexidade do processo de seguimento do perfil e o número de variáveis ligadas a seu desempenho tornam necessário o uso de recursos de simulação e otimização para conseguir desempenhos satisfatórios.

O modelo virtual desenvolvido apresentou desempenho satisfatório para a simulação e otimização da força de interação entre o disco e o perfil. 
O modelo proposto simula adequadamente o comportamento dinâmico de corpo rígido do cortador de base e pode ser usado para efeitos de simulação e aprimoramento do processo de seguimento, em que as variáveis de projeto são aquelas que caracterizam o mecanismo de quatro barras rígidas com suspensão por mola e amortecedor, incluindo uma lei de comportamento mecânicoelástico do contato entre o disco e o perfil.

\section{REFERÊNCIAS}

BRAUNBECK, O.A. Proposta brasileira de colheita mecanizada. In: SEMINÁRIO SOBRE SOLUÇÕES E NOVIDADES NA MECANIZAÇÃO DA CANA-DE-AÇÚCAR, 23., 1999, Ribeirão Preto. Anais... 1999. p.56-60.

DOUGTH, S. Mechanics of machines. New York: John Wiley \& Sons, 1988. 320 p.

MURCIA, G.C. Dimensionamento otimizado de mecanismos articulados para o seguimento do perfil do solo em processos de colheita. 1998. 102 f. Tese (Doutorado em Máquinas Agrícolas) Universidade Estadual de Campinas, Faculdade de Engenharia Agrícola, Campinas 1998.

PASQUINI JÚNIOR, J.; COSTA NETO, A. Determinação das características de handling de veículos automotivos. In: ENCONTRO DE USUÁRIOS DO PROGRAMA ADAMS, 15., 1996, São Paulo. Anais... São Paulo: USP, 1996. p.13-17.

SANTOS, I.F. Dinâmica de sistemas mecânicos: modelagem, simulação, visualização e verificação. São Paulo: Ed. Makron Books, 2000. 272 p. 